INPLASY

PROTOCOL

To cite: Xiang et al.

Comparison of femoral neck system and cannulated screw of femoral neck fractures - a meta-analysis and systematic review. Inplasy protocol

202190070. doi:

10.37766/inplasy2021.9.0070

Received: 22 September 2021

Published: 22 September 2021

Corresponding author:

Zhou Xiang

xiangzhou13@hotmail.com

Author Affiliation:

West China Hospital, Sichuan

University.

Support: National Natural

Science Found.

Review Stage at time of this submission: Preliminary

searches.

Conflicts of interest:

None declared.

\section{Comparison of femoral neck system and cannulated screw of femoral neck fractures - a meta-analysis and systematic review}

Xiang, Z1; Chen, Z2; Luo, R3; Yang, Y4.

Review question / Objective: Compare the clinical efficacy and complications of FNS with cannulated screws for the treatment of femoral neck fractures in adults.

Condition being studied: Several studies have investigated the clinical efficacy and complications of FNS when compared with cannulated screw. But the sample size of each study was relatively small, and there were some differences in the results. Moreover, there is still no meta-analysis to compare the clinical efficacy and complications of FNS with cannulated screws for the treatment of femoral neck fractures in adults. Eligibility criteria: Studies reported data on femoral neck fracture patients; studies compared surgical outcomes femoral neck fractures using FNS with cannulated screw; studies reported data on clinical outcomes or complications.

INPLASY registration number: This protocol was registered with the International Platform of Registered Systematic Review and Meta-Analysis Protocols (INPLASY) on 22 September 2021 and was last updated on 22 September 2021 (registration number INPLASY202190070).

\section{INTRODUCTION}

Review question / Objective: Compare the clinical efficacy and complications of FNS with cannulated screws for the treatment of femoral neck fractures in adults.
Condition being studied: Several studies have investigated the clinical efficacy and complications of FNS when compared with cannulated screw. But the sample size of each study was relatively small, and there 
were some differences in the results. Moreover, there is still no meta-analysis to compare the clinical efficacy and complications of FNS with cannulated screws for the treatment of femoral neck fractures in adults.

\section{METHODS}

Participant or population: Patients with femoral neck fracture.

Intervention: FNS or cannulated screws.

Comparator: Functional results and complications.

Study designs to be included: Case-control study.

Eligibility criteria: Studies reported data on femoral neck fracture patients; studies compared surgical outcomes femoral neck fractures using FNS with cannulated screw; studies reported data on clinical outcomes or complications.

Information sources: Electronic databases.

Main outcome(s): The results of comparison of demographic characteristics, such as sex and age. The results of comparison of clinical outcomes, such as operative time, blood loss, incision length, length of hospital stay and the incidence of complications.

Quality assessment / Risk of bias analysis: Retrospective studies were assessed by Newcastle-Ottawa(NOS), a total of nine scores including the selection, comparability, and outcome were assessed. When the score was greater than 7 points, the quality of the retrospective studies was considered high.

Strategy of data synthesis: Mean differences (MD) with a 95\% confidence interval (Cl) were calculated using the inverse variance method for continuous variables; and risk ratio (RR) with a $95 \% \mathrm{Cl}$ were calculated using the Mantel-Haenszel analysis method for dichotomous variables. In consideration of the Between-studies heterogeneity, the random-effects model was used for meta-analysis.

Subgroup analysis: Subgroup analysis was used for studies stratified by age; regions; type of fracture; Newcastle-Ottawa Scale components.

Sensitivity analysis: Sensitivity analysis was conducted to assess the robustness of the outcomes by sequentially excluding individual studies.

Country(ies) involved: China.

Keywords: Femoral neck fracture; Femoral neck system; cannulated screw; Metaanalysis.

Contributions of each author:

Author 1 - Zhou Xiang.

Author 2 - Zhao Chen.

Author 3 - Rong Luo.

Author 4 - Yun Yang. 